\title{
The global positioning system, relativity, and extraterrestrial navigation
}

\author{
Neil Ashby ${ }^{1}$ and Robert A. Nelson ${ }^{2}$ \\ ${ }^{1}$ Dept. of Physics, University of Colorado \\ Boulder, CO 80309-0390 USA \\ National Institute of Standards \& Technology Affiliate \\ email: ashby@boulder.nist.gov \\ ${ }^{2}$ Satellite Engineering Research Corporation \\ 7710 Woodmont Ave., Suite 1109, Bethesda, MD 20814 USA \\ email: robtnelson@aol.com
}

\begin{abstract}
Relativistic effects play an important role in the performance of the Global Positioning System (GPS) and in world-wide time comparisons. The GPS has provided a model for algorithms that take relativistic effects into account. In the future exploration of space, analogous considerations will be necessary for the dissemination of time and for navigation. We discuss relativistic effects that are important for a navigation system such as at Mars. We describe relativistic principles and effects that are essential for navigation systems, and apply them to navigation satellites carrying atomic clocks in orbit about Mars, and time transfer between Mars and Earth. It is shown that, as in the GPS, relativistic effects are not negligible.
\end{abstract}

Keywords. relativity, navigation, reference systems, time, GPS

\section{Introduction: relativity principles in the GPS}

The earth and its satellites are in free fall. The principle of equivalence implies that over some region near earth's center of mass, the gravitational field strength due to external bodies is cancelled by an apparent equal but opposite field arising from acceleration. Studies of this cancellation have shown that, when transforming from barycentric coordinates to local inertial coordinates, the source of the cancellation is a time derivative of the synchronization term in the time transformation (Ashby, N. \& Bertotti, B. (1986), Nelson, R. A. (1987)). In the local freely falling frame external bodies can be ignored approximately. A single coordinate time variable occurs in the scalar invariant $d s^{2}$. Coordinate time has the property that an event occurs at a unique coordinate time for all observers.

The basis for computation of relativistic effects in the GPS is the following expression for $d s^{2}$ :

$$
d s^{2}=g_{\mu \nu} d x^{\mu} d x^{\nu}=-\left(1+\frac{2 V}{c^{2}}\right)(c d t)^{2}+\left(1-\frac{2 V}{c^{2}}\right)\left(d x^{2}+d y^{2}+d z^{2}\right) .
$$

Here there are no PPN parameters; the spatial coordinates are isotropic; only leading terms of order $c^{-2}$ are kept; the effects of external bodies are neglected, and the potential of the earth $V$ is modelled keeping only monopole and quadrupole terms. Even with these simplifications, numerous relativistic concepts must be employed in understanding the GPS. These include time dilation, gravitational frequency shifts, the Sagnac effect, constancy of the speed of light, the principle of equivalence, relativity of simultaneity, coordinate speed of light, and local inertial frames. 
The proper time elapsed on an atomic clock depends on the clock's history. The increment of proper time may be computed from the scalar $d s$;

$$
d(c \tau)=d(c t) \sqrt{1+\frac{2 V}{c^{2}}-\left(1-\frac{2 V}{c^{2}}\right) \frac{d x^{2}+d y^{2}+d z^{2}}{c^{2} d t^{2}}} \approx\left(1+\frac{V}{c^{2}}-\frac{v^{2}}{2 c^{2}}\right) d(c t) .
$$

In the GPS this result is exploited to synchronize atomic clocks to coordinate time by tracking the clock's coordinate time in terms of the proper time:

$$
\Delta t=\int_{\text {path }} d \tau\left(1-\frac{V}{c^{2}}+\frac{v^{2}}{2 c^{2}}\right) .
$$

When the potential is due only to the earth, the coordinate time is very close to Geocentric Coordinate Time (TCG). For a clock at rest on earth's surface, the gravitational potential is approximately:

$$
V=-\frac{G M}{r}\left(1-\frac{J_{2} a_{1}^{2}}{2 r^{2}}\left(3 \cos ^{2} \theta-1\right)\right)
$$

and the coordinate time increment will be

$$
d t=d \tau\left(1+\frac{G M}{c^{2} r}\left(1-\frac{J_{2} a_{1}^{2}}{2 r^{2}}\left(3 \cos ^{2} \theta-1\right)\right)+\frac{\omega^{2} r^{2} \sin ^{2} \theta}{2 c^{2}}\right),
$$

where $G M=3.986004418 \times 10^{14} \mathrm{~m}^{3} / \mathrm{s}^{2} ; a_{1}=6.378137 \times 10^{6} \mathrm{~m}$ is earth's equatorial radius, $J_{2}=1.08268 \times 10^{-3}$ is the quadrupole moment coefficient, $\omega=7.292115 \times 10^{-5} \mathrm{~s}^{-1}$ and $\theta$ is the the geocentric colatitude, measured down from the north pole. Here we quote values of the constants that define the WGS-84 system, the basis for navigation in the GPS.

The coefficient of $d \tau$ in Eq. (1.5) has very nearly a constant value on earth's geoid, a surface of constant effective potential in the rotating frame in which the last term in Eq. (1.5) contributes a centripetal potential. The constant can be evaluated on the equator, and the result is

$$
\begin{aligned}
\frac{G M}{c^{2} a_{1}} & +\frac{G M J_{2}}{2 c^{2} a_{1}}+\frac{\omega^{2} a_{1}^{2}}{2 c^{2}} \\
& =(6.95349+.00376+.01203) \times 10^{-10}=6.96928 \times 10^{-10} .
\end{aligned}
$$

\section{GPS time}

The constant estimated in Eq. (1.6) is, to within the limitations of the model potential, the same as the defined constant $L_{G}=W_{0} / c^{2}=6.969290134 \times 10^{-10}$ that gives the rate change between TCG and TAI:

$$
d\left(t_{\mathrm{TAI}}\right)=d\left(t_{\mathrm{TCG}}\right)\left(1-L_{G}\right) .
$$

Noise on individual clocks can be mitigated by averaging over many clocks; Eq. (2.1) can be interpreted as a definition of the TCG time scale in terms of TAI, which is an averaged time scale maintained by the BIPM that incorporates hundreds of atomic clocks distributed around the world. The USNO maintains a time scale, UTC(USNO), based on its own ensemble of atomic clocks, and is a major contributor to TAI (or terrestrial time TT). To change the coordinate time $t$ of Eq. (1.2) so that it represents TT, let $t \rightarrow t_{\mathrm{TT}} /\left(1-L_{G}\right)$. Solving to leading order in $c^{-2}$ for the elapsed coordinate time with 
the new scale,

$$
\Delta t_{\mathrm{TT}}=\int_{\text {path }} d \tau\left(1-\frac{V}{c^{2}}-\frac{W_{0}}{c^{2}}+\frac{v^{2}}{2 c^{2}}\right) .
$$

Clocks in GPS satellites are offset so that they beat at the same rate as the references on earth's geoid; also the altitude is so great that the orbits are nearly Keplerian, earth's quadrupole potential being very small. For such clocks, conservation of energy gives

$$
\frac{1}{2} v^{2}-\frac{G M}{r}=-\frac{G M}{2 a},
$$

where $a$ is the semi-major axis. Combining Eqs. (2.2-2.3) and rearranging,

$$
\begin{aligned}
& \Delta t=\int_{\text {path }} d \tau\left(1+\frac{3 G M}{2 a c^{2}}-\frac{W_{0}}{c^{2}}+\frac{2 G M}{c^{2}}\left(\frac{1}{a}-\frac{1}{r}\right)\right) \\
= & \Delta \tau\left(1-4.4647 \times 10^{-10}\right)+\frac{2 \sqrt{G M a}}{c^{2}} e \sin E+\text { const }
\end{aligned}
$$

where $E$ is the eccentric anomaly. The constant rate offset, $-4.4647 \times 10^{-10}$ contains a handful of relativistic corrections:

$$
\frac{3 G M}{2 a c^{2}}-\frac{W_{0}}{c^{2}}=-4.4647 \times 10^{-10} .
$$

GPS satellite clocks are given this offset before launch so that in orbit, they will beat at the correct coordinate rate. GPS clocks are steered (without leap seconds) to the UTC(USNO) time scale so they provide a realization of the coordinate time scale TT, except that the last term in Eq. (2.4) must be implemented in all GPS receivers.

A mysterious "break" in satellite clock frequencies occurred when satellite orbits were adjusted, moving them up or down between their assigned slots and parking orbits. This was explained in about 2000 in terms of changes in the satellite semimajor axis:

$$
\delta\left(\frac{3 G M}{2 c^{2} a}\right) \approx-\frac{3 G M \delta a}{2 c^{2} a^{2}} .
$$

This term is a combination of time dilation and gravitational frequency shifts. A change of $20 \mathrm{~km}$ in the semi-major axis results in a frequency change of a few parts in $10^{13}$. This correction is currently implemented by hand in the GPS.

\section{Earth rotation and the Sagnac effect}

To be useful for navigation, GPS clocks are synchronized in a freely falling, locally inertial frame (the ECI frame) whose origin is at earth's center of mass. If earth rotation were ignored when synchronizing clocks on earth's surface, the results would be inconsistent because light does not travel in a straight line with uniform speed $c$ in an earth-fixed, rotating reference frame. If we ignore the gravitational potential, the metric of special relativity in cylindrical coordinates is

$$
d s^{2}=-(c d t)^{2}+d r^{2}+r^{2} d \phi^{2}+d z^{2} .
$$

If we transform to an earth-centered, earth-fixed (ECEF) reference frame by making the replacement

$$
\phi \rightarrow \phi+\omega t
$$


where $\omega$ is earth's angular velocity of rotation, then the metric becomes

$$
d s^{2}=-\left(1-\frac{\omega^{2} r^{2}}{c^{2}}\right)\left(c^{2} d t^{2}\right)+d r^{2}+2 \omega r^{2} d \phi d(c t)+d z^{2} .
$$

The coordinate time is still $t$ (or effectively TT). Solving for $d t$ to leading order in $\omega$ for a slowly moving clock,

$$
\Delta t=\int_{\text {path }} d \tau+\frac{2 \omega}{c^{2}} \int_{\text {path }} d A_{z}
$$

where $A_{z}$ is the area swept out by a vector from the rotation axis to the clock, projected onto a plane parallel to earth's equator. This correction can be several hundred nanoseconds and is an important correction that must be accounted for in long-distance clock comparisons such as when using GPS in common view from laboratories in Europe and the U.S., or in TWSTFT (two-way satellite time and frequency transfer). The same effect occurs if electromagnetic signals are used to synchronize clocks.

\section{Navigation with the GPS}

We henceforth assume that clocks in GPS satellites are synchronized (or equivalently, that their biases are known). Suppose that at coordinate time $t$ a receiver at position $\mathbf{r}$ detects time ticks originating at times $t_{j}$ from satellites at positions $\mathbf{r}_{j}$. The navigation equations are then

$$
c\left(t-t_{j}\right)=\left|\mathbf{r}-\mathbf{r}_{j}\right|, \quad j=1,2,3,4 \ldots
$$

Given four such equations, in principle these non-linear equations can be solved for the unknown time $t$ and position $\mathbf{r}$ of the receiver's detection event. However, the GPS is designed so that the satellite positions are transmitted in the ECEF! This is a source of much confusion. The satellite positions must be transformed to some common ECI frame before solving, then the solution transformed to the ECEF frame that exists at time $t$.

The basic measurement of the detector is accomplished by alignment of a code unique to each transmitter, that is impressed on the signal by phase reversals, with a replica of the code generated within the receiver. The receiver carries a relatively inexpensive (and noisy) oscillator for code generation and timing. Suppose the clock in the receiver has an error $\Delta t_{r}$. The time difference between transmitted time $t_{j}$ and time $t+\Delta t_{r}$ on the receiver oscillator is called the "pseudorange." Then the pseudorange $P R_{j}$ is

$$
P R_{j}=c\left(t+\Delta t_{r}-t_{j}\right)=\sqrt{\left(x-x_{j}\right)^{2}+\left(y-y_{j}\right)^{2}+\left(z-z_{j}\right)^{2}}+c\left(\Delta t_{r}-\Delta t_{t}+\Delta t_{\mathrm{rel}}+\Delta t_{\mathrm{atm}}\right),
$$

where $\Delta t_{\mathrm{rel}}$ is the eccentricity correction, $\Delta t_{t}$ is the transmitter error, and $\Delta t_{\mathrm{atm}}$ are atmospheric delay corrections. This is illustrated in Figure 1.

The time bias on the oscillator is continually being recalibrated to within a few nanoseconds by internal solutions of the navigation equations, but inexpensive receivers do not make such precision available to the user.

Signals from the GPS satellites are right circularly polarized, in which the electric and magnetic fields oscillate in phase. At places in the wave train where a phase reversal is imposed, all electromagnetic fields will pass through zero. At a physical point where all fields are zero, the fields are zero in every reference system; these invariant zeros sweep through free space with speed $c$. 


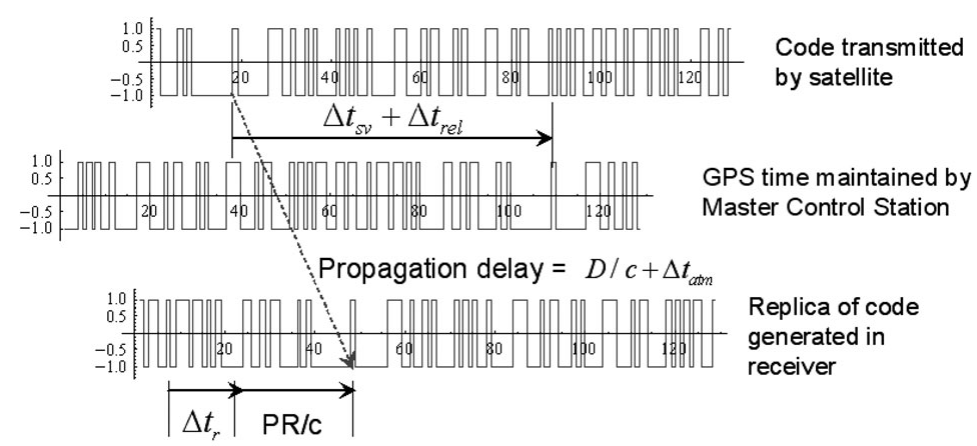

Figure 1. Pseudorange is the true range $D$ with corrections due to relativity, clock biases, and atmospheric delays. The carrier is not shown; the plotted code corresponds to phase reversals impressed on the carrier.

\section{Other satellite navigation systems}

Several other navigation systems have been partially deployed or are in various stages of planning. The Russion GLONASS system is very similar to GPS. Satellites orbit 17 times while GPS orbits 16 times. A clock frequency offset, which is not quite as large as that for GPS, is applied to the satellite clocks. A full constellation of 24 satellites was planned but was never completely implemented; currently the constellation is being replenished and a full constellation is in the works.

GALILEO is a European satellite navigation system which at present has two satellites in orbit. Specifications for this system call for all relativistic effects to be the responsibility of the receiver. No frequency offsets will be applied in hardware to the orbiting clocks, which will necessarily run faster than TT by a few parts in $10^{10}$. It will be up to software in the receiver to correct the transmitted time ticks for this large time drift.

The Chinese Republic has plans for a global satellite navigation system involving over 30 satellites; some of these will be geosynchronous and more than one such satellite has already been launched.

Several augmentation systems such as WAAS, EGNOS, and QZSS monitor GPS signals and upload data to geostationary satellites for retransmission to users. This mitigates problems with GPS due to outages, multipath, ionospheric delays, and other problems that make GPS somewhat unreliable.

\section{Effects not currently accounted for}

Solar and lunar tidal potentials. At the origin of a locally inertial, freely falling reference frame such as the ECI frame, the principle of equivalence implies that the gravitational field strength due to external bodies is cancelled by the induced field strength due to acceleration. Such a cancellation has been overlooked time and again by no small number of individuals claiming that relativity has not been accounted for properly in the GPS. The cancellation is a consequence of the relativity of simultaneity. To summarize the calculation briefly, consider a time transformation from barycentric or heliocentric coordinates to a locally inertial, freely falling frame, that has a resynchronization term of the form

$$
\frac{\mathbf{v} \cdot \mathbf{r}}{c^{2}}
$$

where $\mathbf{v}$ is the velocity of the local frame's origin and $\mathbf{r}$ is the displacement of the observation point from the origin. The tensor transformation of the 00-component of the 
metric tensor will then produce contributions including terms

$$
-\left(1+\frac{\partial \mathbf{v} \cdot \mathbf{r} / c^{2}}{\partial t}\right)^{2}=-\frac{2 \mathbf{a} \cdot \mathbf{r}}{c^{2}}+\ldots
$$

where $\mathbf{a}$ is the acceleration of the freely falling frame. Let the contributions to $g_{00}$ of external bodies be expanded in a Taylor series about the origin of the local frame. The first term will be the same for all clocks in the neighborhood of the local frame origin and can be removed by absorbing the term into the local time scale. The second term will be $-2 \nabla V \cdot \mathbf{r} / c^{2}$ and these two terms together will be

$$
-\frac{2}{c^{2}}(\mathbf{a}+\nabla V) \cdot \mathbf{r}=0
$$

because in free fall, $\mathbf{a}=-\nabla V$. The cancellation does not arise from second-order Doppler effects as some have claimed. The first terms that contribute are tidal terms, second derivatives of the external potential evaluated at the origin of the local frame. The net fractional frequency shift of orbiting GPS clocks due to the moon has an amplitude of only $7 \times 10^{-16}$ and the sun contributes about half that amount.

Earth's quadrupole potential. Earth's quadrupole moment produces a small, predictable periodic effect on orbiting clocks, that is not currently accounted for. The time correction that should be applied to the orbiting clock is (Ashby (2003))

$$
\Delta t_{\text {oblateness }}=\frac{G M J_{2} a_{1}^{2}}{2 c^{2} a^{3}}\left(1-\frac{3}{2} \sin ^{2} i\right) \Delta \tau+\sqrt{\frac{G M}{a^{3}}} \frac{J_{2} a_{1}^{2} \sin ^{2} i}{2 c^{2}} \sin (2 \omega+2 f) .
$$

where $i$ is the orbital inclination, $\omega$ is the altitude of perigee, $f$ is the satellite's true anomaly, and $a$ is the mean semi-major axis. It is coincidental that the chosen inclination $i=55^{\circ}$ so that the secular term is nearly zero. The remaining term has a period of nearly 6 hours and an amplitude of 24 ps, contributing almost a centimeter to navigation error.

Coordinate speed of light. For signals traveling from earth's surface at radius $R$ to or from a GPS satellite, at an elevation angle $E$, solving the equation of the null geodesic $d s^{2}=0$ for the propagation time gives

$$
\begin{array}{r}
c \Delta t=\left(1-L_{G}\right)\left(\sqrt{r_{S V}^{2}-R^{2} \cos ^{2} E}-R \sin E\right)+ \\
\frac{2 G M}{c^{2}} \log \frac{r_{S V}+R+\sqrt{r_{S V}^{2}-R^{2} \cos ^{2} E}-R}{r_{S V}+R-\sqrt{r_{S V}^{2}-R^{2} \cos ^{2} E}+R},
\end{array}
$$

where $r_{S V}$ is the satellite's radial coordinate. The correction factor $L_{G}$ multiplying the geometric range very nearly cancels the usual logarithmic Shapiro delay term (Petit \& Wolf (1994)). The isotropic coordinate gauge has been selected and the product $G M$ for earth is determined as part of the definition of the WGS-84 reference frame used for navigation in the GPS. Also, $c$ is defined so $G M / c^{2}$ has fixed units of length. Further scaling of the length coordinate would be inconsistent since the first-order potential term $G M /\left(c^{2} r\right)$ is unitless and at a given physical point must have the same numerical value in all coordinate systems. Figure 1 plots both corrections to the delay.

\section{Extension to extraterrestrial navigation}

Consider extending the analysis of relativistic effects to the vicinity of another celestial body such as Mars (Ares) (Nelson (2007)). An equipotential surface exists in the Marscentered, Mars-fixed rotating frame (ACAF frame) in which atomic clocks at rest beat 


\section{Correction for gravitational time delay}

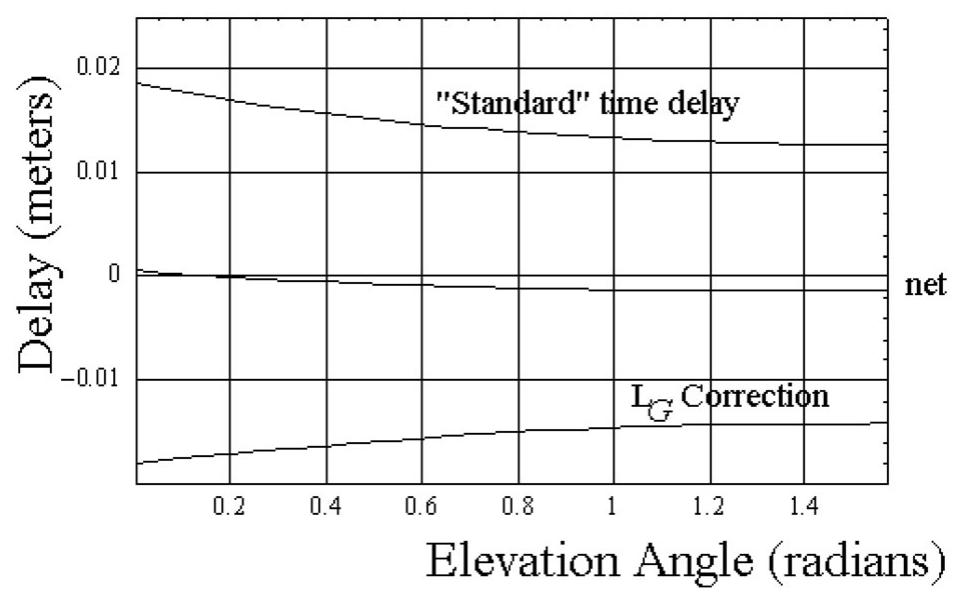

Figure 2. Plot of relativistic corrections to coordinate time delay. The log term is nearly cancelled by the $L_{G}$ correction to the geometric distance.

at the same rate. Ignoring all masses except Mars, the rate of Aretian coordinate time relative to clocks at "infinity" may be expressed in terms of a constant like $L_{G}$.

$$
\begin{aligned}
& L_{A}=\frac{G M_{A}}{c^{2} a_{1 A}}+\frac{G M_{A} J_{2 A}}{2 c^{2} a_{1 A}}+\frac{\omega_{A}^{2} a_{1 A}^{2}}{2 c^{2}} \\
\approx & (1.40362+.00128+.00322) \times 10^{-10} \\
= & 1.40812 \times 10^{-10} \quad\left(21 \% \text { of } L_{G}\right) .
\end{aligned}
$$

Then the frequency offset of an atomic clock in orbit around Ares can be calculated when the semimajor axis is known. For a circular orbit,

$$
\frac{\Delta f}{f}=\frac{3 G M_{A}}{2 a_{A} c^{2}}-L_{A} .
$$

For an areosynchronous orbit, $a_{A}=2.04277 \times 10^{7} \mathrm{~km}$, and

$$
\frac{\Delta f}{f}=-1.05738 \times 10^{-10} \text {. }
$$

Figure 3 shows an areosynchronous navigation system poised over a possible base near the Arean equator. The satellites are given slightly inclined orbits; five satellites are needed in order to provide continuous navigation coverage. The reason for this can be visualized as follows. Think of the navigation error associated with signals from one satellite in terms of a narrow space between two concentric shells of nearly equal radii. Two such shells that intersect in general give a thin torus of position, but if the shells happen to be tangent such a torus spreads into a disc and the position is not well determined. This can happen if the centers of the shells are in line with the point of tangency, and this is the reason that poor navigation precision could result if all the satellites were in the equatorial plane. Imagine any two of the five satellites, not in equatorial orbits. The plane generated by vectors from the center of Ares to the satellites will be wobbling in space and will eventually intersect one of the remaining satellites. When that happens, with 


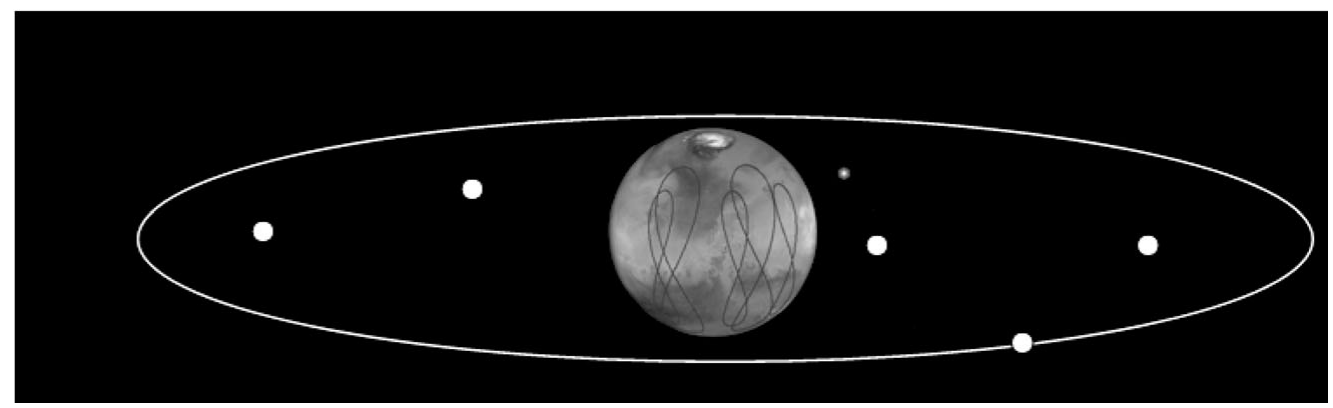

Figure 3. Notional areosynchronous navigation system with five satellites in slightly inclined orbits

three satellites in line, the geometrical situation is poor for navigation; one of the three satellites is relatively useless; in general one needs five satellites.

For continuous communication coverage between the Arean surface and the satellites, twelve satellites distributed in three orbital planes will suffice; however such a configuration is not sufficient for navigation, where continuous view of at least four satellites from points on the surface are needed. A navigational system with 24 satellites similar to the GPS configuration would be visible most of the time at a radius of about 4.2 planetary radii: if $a_{A}=14,259 \mathrm{~km}$, the orbital period would be $51,695 \mathrm{~s}$, and the fractional frequency offset relative to clocks on the surface would be

$$
\frac{3 G M_{A}}{2 c^{2} a_{A}}-L_{A}=1.90941 \times 10^{-10} .
$$

Barycentric Coordinate Time (TCB). Time transfer between an extraterrestrial time system and a terrestrial one calls for a common time system which overlaps both systems. We consider here TCB according to the IAU definitions (IAU/IUGG 1991). The elapsed Barycentric Coordinate Time on a moving clock anywhere within the solar system is

$$
\Delta t_{\mathrm{TCB}}=\int_{\text {path }} d \tau\left(1-\frac{V(\mathbf{r})}{c^{2}}+\frac{1}{2} \frac{v^{2}}{c^{2}}\right),
$$

where $\mathbf{r}$ is the position of the clock and $v$ its velocity. All solar system bodies are included in the potential $V(\mathbf{r})$. To obtain terrestrial time in terms of TCB, The vector $\mathbf{r}$ and the corresponding velocity are split up into into vectors from the barycenter to earth, and relative to earth:

$$
\mathbf{r}=\mathbf{r}_{\text {earth }}+\mathbf{R}, \quad \mathbf{v}=\mathbf{v}_{\text {earth }}+\dot{\mathbf{R}} .
$$

where $\mathbf{R}$ is the displacement of the clock relative to earth's center of mass. The potential is split into a contribution from the earth and an external potential that is expanded in a Taylor series for small displacements:

$$
V=V_{\text {earth }}+V_{\text {ext }} \approx V_{\text {earth }}(\mathbf{R})+V_{\text {ext }}\left(\mathbf{r}_{\text {earth }}\right)+\nabla V_{\text {ext }} \cdot \mathbf{R}+\ldots
$$

Then after substitution and rearrangement, and replacing the integration variable $\tau$ by $t$ in first-order correction terms,

$$
\begin{gathered}
\Delta t_{\mathrm{TCB}}=\Delta \tau+\int_{t_{0}}^{t_{1}} d t\left(-\frac{V_{\text {ext }}\left(\mathbf{r}_{\text {earth }}\right)}{c^{2}}+\frac{1}{2} \frac{v_{\text {earth }}^{2}}{c^{2}}\right) \\
+\int_{t_{0}}^{t_{1}} d t\left(-\frac{V_{\text {earth }}(\mathbf{R})}{c^{2}}+\frac{1}{2} \frac{\dot{\mathbf{R}}^{2}}{c^{2}}\right)+\left.\frac{\mathbf{R} \cdot \mathbf{v}_{\text {earth }}}{c^{2}}\right|_{t_{0}} ^{t_{1}} .
\end{gathered}
$$


The last term in Eq. (7.8) represents the relativity of simultaneity of earth's ECI frame moving relative to the barycenter. Only tidal terms from the external potential survive, because of the principle of equivalence. The first integral in Eq. (7.8) gives hundreds of correction terms; the principal ones are similar to the corrections discussed in Eq. (2.4) for a GPS clock:

$$
\int_{t_{0}}^{t_{1}} d t\left(-\frac{V_{\text {ext }}\left(\mathbf{r}_{\text {earth }}\right)}{c^{2}}+\frac{1}{2} \frac{v_{\text {earth }}^{2}}{c^{2}}\right) \approx \frac{3 G M_{\odot}}{2 c^{2} a_{\text {earth }}}+\frac{2 \sqrt{G M_{\odot} a_{\text {earth }}}}{c^{2}} e_{\text {earth }} \sin E_{\text {earth }}+\ldots
$$

The second integral in Eq. (7.8) depends on the position and velocity of the clock relative to the earth, and is just the TCG of the event:

$$
\int_{t_{0}}^{t_{1}} d t\left(-\frac{V_{\text {earth }}(\mathbf{R})}{c^{2}}+\frac{1}{2} \frac{\dot{\mathbf{R}}^{2}}{c^{2}}\right)=t_{\mathrm{TCG}}=\left(1+L_{G}\right) t_{\mathrm{TT}} .
$$

The relation between Arean time and TCB is analogous. The time of an event near Ares can then be transformed to TCB and thence to TT.

\section{Conclusions}

Numerous relativistic effects must be accounted for in global navigation systems. A distributed network of clocks, synchronized in the ECI frame, provides a realization of coordinate time used for many purposes other than simple navigation. Trends toward improving the precision of navigation algorithms will entail the incorporation of additional relativistic effects.

Clocks on GPS satellites would run fast by 38 microseconds per day relative to terrestrial clocks if they were not offset prior to launch. Also, the navigation solution in the GPS could be in error if the residual effects of time dilation and gravitational redshift due to orbit eccentricity were not corrected in the receiver (14 meters error if $e=0.02$ ).

Similarly, the times registered by clocks on Mars will require relativistic corrections. If signals from a clock on the planetary surface were used for navigation by an approaching spacecraft, then serious errors would result if the physics of relativity were not considered. Also, any precisely timed astronomical event measured from the vicinity of the planet must be calibrated correctly in terms of the times on Earth, and the gravitational delay in the propagation of an electromagnetic signal must be considered.

The fundamental notion is that time is the reading of a clock. All time comparisons are made between clocks, whose readings represent "proper time." In any coordinate system, the relation between coordinate time and proper time is given by the invariant spacetime interval. The choice of coordinate time is arbitrary and is based on convenience.

The paradigm for relativistic time transfer has been successfully applied in the GPS. It is important that analogous relativistic effects be recognized in the synchronization of clocks for future applications in the exploration of the solar system. They are not merely of theoretical scientific interest. Relativity has entered into the realm of engineering practice.

\section{References}

Ashby, N. \& Bertotti, B. 1986 Phys. Rev., D34, 2246-2258

Nelson, R. A. 1987 J. Math. Phys., 28, 2379-2383; ibid. 35, 6224-6225

Ashby, N. 2003 http://relativity.livingreviews.org/Articles/lrr-2003-1

Ashby, N. 2002 Phys. Today, 55(5) 41-47

Petit, G. \& Wolf, P. 1994 Astron. Astrophys. 286, 971-977

Nelson, R. A. 2007 "Relativistic Time Transfer in the Solar System", Proc. EFTF/IEEE-FCS, Geneva, 1278-1283 